\title{
Lee waves in a stratified flow \\ Part 3. Semi-elliptical obstacle
}

\section{By HERBERT E. HUPPERT $\uparrow$ AND JOHN W. MILES $\uparrow$}

\author{
Institute of Geophysics and Planetary Physics \\ University of California, La Jolla
}

(Received 9 May 1968)

The stratified shear flow over a two-dimensional obstacle of semi-elliptical crosssection is considered. The shear flow is assumed to be inviscid with constant upstream values of the density gradient and dynamic pressure (Long's model). Two complete sets of lee-wave functions, each of which satisfies the condition of no upstream reflexion, are determined in elliptic co-ordinates for $\epsilon \geqslant 1$ and $\epsilon \leqslant 1$, where $\epsilon$ is the ratio of height to half-width of the obstacle. These functions are used to determine the lee-wave field produced by, and the consequent drag on, a semi-elliptical obstacle as functions of $\epsilon$ and the reduced frequency (reciprocal Froude number) within the range of stable flow. The reduced frequency at which static instability first occurs is calculated as a function of $\epsilon$.

\section{Introduction}

We consider the generation of lee waves by, the consequent wave-drag on, and the parametric range of (statically) stable flow for a two-dimensional obstacle of semi-elliptical cross-section in a stratified shear flow. We assume that the flow is inviscid and steady and that the upstream density gradient and dynamic pressure are constant (Long's model) and obtain a solution by separation of variables. The present paper is a natural extension of parts 1 and 2 (Miles $1968 a, b)$, in which an infinitely thin barrier and a semi-circle are considered. We obtain approximate solutions for obstacles of more general shape in part 4 (Miles \& Huppert 1969) and test these approximate solutions by comparing those for a semi-elliptical obstacle with the exact solutions obtained here. We refer subsequently to the various parts as I, II and IV followed by the appropriate section or equation number.

Let $H$ represent the height of the ellipse, $B$ its half-width and $U, \rho$, and $N$ the wind speed, density, and intrinsic (Väisälä) frequency of the basic flow. The hypotheses that the upstream dynamic pressure,

$$
q=\frac{1}{2} \rho U^{2}
$$

and the upstream density gradient are constant imply (Long 1953) that the vertical displacement of a streamline, say $H \delta$, from its position in the basic flow satisfies the Helmholtz equation

$$
\nabla^{2} \delta+\kappa^{2} \delta=0,
$$

$\dagger$ Also Department of Aerospace and Mechanical Engineering Sciences. 
where

$$
\kappa=N H / U
$$

is the reduced frequency (reciprocal Froude number) and $x, y$ are dimensionless, Cartesian co-ordinates based on $H$ (see figure 1). We satisfy the boundary condition of zero normal velocity at the obstacle surface, say $C$, and the hypothesis of no upstream reflexion of waves by setting

$$
\begin{aligned}
& \delta=0 \quad\left(y=0, \quad|x|>\epsilon^{-1}\right), \\
& \delta=y \quad \text { on } C \text {, } \\
& \delta=o\left(r^{-\frac{1}{2}}\right) \quad(x \rightarrow-\infty), \\
& \epsilon=H / B \text {. }
\end{aligned}
$$

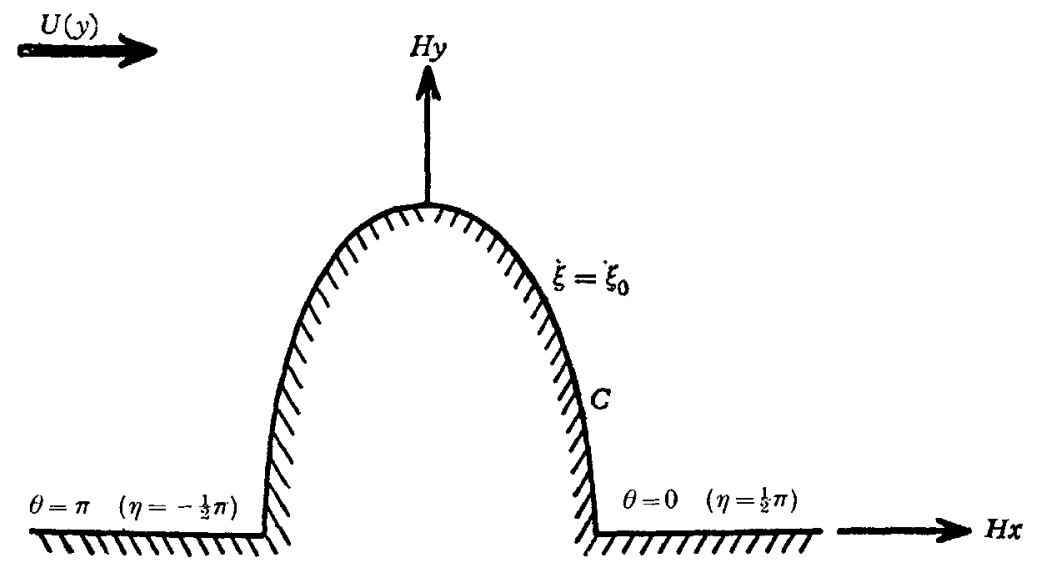

FIGURE 1. Geometrical configuration for the tall ellipses. The configuration is similar for the squat ellipses except that $\eta=0(\pi)$ along the positive (negative) $x$-axis.

As in II, we pose the asymptotic representation of the solution to (1.2) and (1.4) in the form

$$
\delta \sim(2 / \pi \kappa r)^{\frac{1}{2}} \mathscr{R}\left\{\exp \left[i\left(\kappa r-\frac{1}{4} \pi\right)\right] f(\theta)\right\} \quad(\kappa r \rightarrow \infty, \quad x>0),
$$

where $f(\theta)$ is the complex scattering amplitude of the far field. We define [ef. II (1.8)-(1.10)] the differential scattering cross-section, $\sigma(\theta)$, and the total scattering cross-section, $Q$, and relate these to the wave-drag on the obstacle, $D$, by

$$
\begin{aligned}
\sigma(\theta) & =(2 H \kappa / \pi)|f(\theta)|^{2} \\
Q & =\int_{0}^{\frac{1}{2} \pi} \sigma(\theta) d \theta \\
D & =q \int_{0}^{\frac{1}{2} \pi} \sigma(\theta) \cos \theta d \theta .
\end{aligned}
$$

We determine these three quantities as functions of $\kappa$ and $\epsilon$ for $0<\kappa<\kappa_{c}$, where $\kappa_{e}$ is that value of $\kappa$ for which the condition for static stability, $\delta_{y} \leqslant 1$, is first violated. Local density inversions (which imply static instability) and flow reversals appear for $\kappa>\kappa_{c}$, in consequence of which the basic hypotheses on which Long's model rests are almost certainly untenable (see I for a more com- 
plete discussion of this point). Closed streamlines appear for $\kappa>\kappa_{1}$, where $\kappa_{1}$ is only slightly greater than $\kappa_{c}$; their existence definitely violates Long's hypothesis that all streamlines originate in the undisturbed, upstream flow.

The analysis falls naturally into two sections: $\epsilon \geqslant 1$, the tall ellipses, and $\epsilon \leqslant 1$, the squat ellipses. The thin barrier corresponds to $\epsilon=\infty$, the semi-circular obstacle to $\epsilon=1$. The squat ellipses correspond more closely to obstacles of physical interest.

We obtain the solution to (1.2) and (1.4) as an expansion in a complete set of functions, each of which satisfies $(1.4 a, c)$. We then determine the expansion coefficients by invoking the boundary condition (1.4b). This leads to an infinite set of coupled, linear equations, which we solve approximately by truncation. The number of equations, say $N$, that must be solved depends upon $\epsilon$. For $\epsilon>1$, $N=1$ leads to reasonably accurate results, and $N=2$ is numerically adequate for $\kappa<\kappa_{c}$. For $\epsilon<1$, we find that $N=3$ suffices except for very small $\epsilon$, say $\epsilon<0 \cdot 1$, for which $N \geqslant 5$ would be required. We present an alternative procedure for determining the solution in the limit $\epsilon \rightarrow 0$ for fixed $\kappa$ in IV.

An alternative approach to the boundary-value problem posed by (1.2) and (1.4) is via the construction of the Green's function (point-source solution) for the half-space. The analysis is similar to that presented in II.

\section{Lee-wave functions for the tall ellipses $(\epsilon \geqslant 1)$}

We define the elliptic co-ordinates $\xi$ and $\eta$ and the modified, reduced frequency $\omega$ by

and

$$
x=h \sinh \xi \sin \eta, \quad y=h \cosh \xi \cos \eta,
$$

$$
\omega=\kappa h=(N H / U)\left\{1-(B / H)^{2}\right\}^{\frac{1}{2}},
$$

where

$$
h=\epsilon^{-1}\left(\epsilon^{2}-1\right)^{\frac{1}{2}}=\left\{1-(B / H)^{2}\right\}^{\frac{1}{2}} .
$$

The obstacle is then specified by the $\xi$-surface

$$
\xi=\xi_{0} \equiv \operatorname{coth}^{-1} \epsilon .
$$

Substituting (2.1) and (2.2) into (1.2) and (1.4) and denoting the displacement function $\delta$ as $\delta(\xi, \eta)$, we obtain

$$
\begin{gathered}
\delta_{\xi \xi}+\delta_{\eta \eta}+\omega^{2}\left(\sinh ^{2} \xi+\sin ^{2} \eta\right) \delta=0, \\
\delta\left(\xi, \pm \frac{1}{2} \pi\right)=0 \quad\left(\xi>\xi_{0}\right), \\
\delta\left(\xi_{0}, \eta\right)=\cos \eta,
\end{gathered}
$$

and

$$
\delta(\xi, \eta)=o\left(\exp \left[-\frac{1}{2} \xi\right]\right) \quad\left(\xi \rightarrow \infty, \quad-\frac{1}{2} \pi<\eta<0\right) .
$$

We seek a set of elliptical lee-wave functions, say $\delta_{n}(\xi, \eta), n=1,2, \ldots$, appropriate to the tall ellipses $(\epsilon \geqslant 1)$ that is complete in the interval $|\eta|<\frac{1}{2} \pi$ for fixed $\xi$ and each member of which satisfies (2.3) and $(2.4 a, c)$. We find that a set satisfying (2.3) and $(2.4 a)$, subsequently referred to as $\mathscr{S}$, is given by

$$
\begin{aligned}
& \delta_{2 n+1}(\xi, \eta)=M c_{2 n+1}^{(2)}\left(\xi, \frac{1}{4} \omega^{2}\right) c e_{2 n+1}\left(\eta, \frac{1}{4} \omega^{2}\right)+\psi_{2 n+1}(\xi, \eta) \quad(n=0,1, \ldots) \\
& \text { and } \quad \delta_{2 n}(\xi, \eta)=M s_{2 n}^{(2)}\left(\xi, \frac{1}{4}\left(\omega^{2}\right) s e_{2 n}\left(\eta, \frac{1}{4} \omega^{2}\right)+\psi_{2 n}(\xi, \eta) \quad(n=1,2, \ldots)\right.
\end{aligned}
$$


where

$$
\begin{gathered}
\psi_{2 n+1}(\xi, \eta)=\sum_{r=1}^{\infty} G_{n r} M s_{2 r}^{(1)}\left(\xi, \frac{1}{4} \omega^{2}\right) s e_{2 r}\left(\eta, \frac{1}{4} \omega^{2}\right) \\
\psi_{2 n}(\xi, \eta)=\sum_{r=0}^{\infty} H_{n r} M c_{2 r+1}^{(1)}\left(\xi, \frac{1}{4} \omega^{2}\right) c e_{2 r+1}\left(\eta, \frac{1}{4} \omega^{2}\right) .
\end{gathered}
$$

and

Our notation for the Mathieu functions follows that of Blanch (1964): $c e_{n}\left(\eta, \frac{1}{4} \omega^{2}\right)$ and $s e_{n}\left(\eta, \frac{1}{4} \omega^{2}\right)$ are periodic Mathieu functions and $M c_{n}^{(j)}\left(\xi, \frac{1}{4} \omega^{2}\right)$ and $M s_{n}^{(j)}\left(\xi, \frac{1}{4} \omega^{2}\right)$ $(j=1,2)$ are radial Mathieu functions; we suppress the parametric argument of these functions, $\frac{1}{4} \omega^{2}$, in the subsequent development.

We can show, using known theorems in Hilbert space, that $\mathscr{S}$ forms a complete set in the interval $|\eta|<\frac{1}{2} \pi$.

Invoking the known, asymptotic expansions of the radial Mathieu functions (loc. cit. ante. p. 740) in (2.5) and (2.6) and requiring the resulting $\delta_{n}$ to satisfy $(2.4 c)$, we find that the coefficients $G_{n r}$ and $H_{n r}$ must be determined such that

and

$$
\begin{gathered}
s e_{2 n}(\eta)=\sum_{r=0}^{\infty}(-)^{n+r+1} H_{n r} c e_{2 r+1}(\eta) \\
c e_{2 n+1}(\eta)=\sum_{r=1}^{\infty}(-)^{n+r} G_{n r} s e_{2 r}(\eta)
\end{gathered}
$$

in $\eta=\left(-\frac{1}{2} \pi, 0\right)$. Invoking the fact that each of the sets $s e_{2 r}(\eta)$ and $c e_{2 r+1}(\eta)$ is orthogonal and complete in $\left(-\frac{1}{2} \pi, 0\right)$, we satisfy $(2.4 c)$ by choosing

and

$$
G_{n r}=-K_{r n}, \quad H_{n r}=K_{n r}
$$

$$
\begin{aligned}
K_{r n} & =(-)^{n+r} 2 \pi^{-1} \int_{0}^{\pi} s e_{2 r}(t) c e_{2 n+1}(t) d t \\
& =(-)^{n+r} 4 \pi^{-1} c e_{2 n+1}(0) s e_{2 r}^{\prime}(0)\left(b_{2 r}-a_{2 n+1}\right)^{-1} \quad(n=0,1, \ldots, r=1,2, \ldots),
\end{aligned}
$$

where $a_{2 n+1}$ and $b_{2 r}$ denote the characteristic values of the even and odd periodic Mathieu functions respectively.

We derive the alternative, integral representations

$$
\psi_{2 n+1}(\xi, \eta)=(-)^{n} \pi^{-1} \int_{0}^{\pi} \sin (\omega \cosh \xi \cos \eta \cos t) \sin (\omega \sinh \xi \sin \eta \sin t) c e_{2 n+1}(t) d t
$$

and

$$
\psi_{2 n}(\xi, \eta)=(-)^{n} \pi^{-1} \int_{0}^{\pi} \sin (\omega \cosh \xi \cos \eta \cos t) \cos (\omega \sinh \xi \sin \eta \sin t) s e_{2 n}(t) d t
$$

by expanding the integrands in $(2.10 a, b)$ in Fourier-Mathieu series and integrating term by term. These integrals are used subsequently to determine the streamlines (the contours of $y-\delta$ ).

Recalling that $c e_{2 n+1}(\eta)\left[s e_{2 r}(\eta)\right]$ are even [odd] functions, we see that asymptotic cancellation of the two terms of $(2.5)$ in $\eta=\left(-\frac{1}{2} \pi, 0\right)$ implies their asymptotic equivalence in $\eta=\left(0, \frac{1}{2} \pi\right)$. Hence, far downstream from the ellipse the leewave functions have the form

$$
\begin{aligned}
\delta_{2 n+1}(\xi, \eta) & \sim 2 M c_{2 n+1}^{(2)}(\xi) c e_{2 n+1}(\eta) \quad\left(\omega \cosh \xi \rightarrow \kappa r \rightarrow \infty, \quad 0 \leqslant \eta<\frac{1}{2} \pi\right) \\
& \sim 2^{\frac{3}{2}}(-)^{n}(\pi \omega \cosh \xi)^{-\frac{1}{2}} \cos \left(\omega \cosh \xi-\frac{1}{4} \pi\right) c e_{2 n+1}(\eta) \quad(\xi \rightarrow \infty),
\end{aligned}
$$


and

$$
\begin{aligned}
\delta_{2 n}(\xi, \eta) & \sim 2 M s_{2 n}^{(2)}(\xi) s e_{2 n}(\eta) \quad\left(\omega \cosh \xi \rightarrow \kappa r \rightarrow \infty, 0 \leqslant \eta<\frac{1}{2} \pi\right) \\
& \sim 2^{\frac{3}{2}}(-)^{n}(\pi \omega \cosh \xi)^{-\frac{1}{2}} \sin \left(\omega \cosh \xi-\frac{1}{4} \pi\right) s e_{2 n}(\eta) \quad(\xi \rightarrow \infty) .
\end{aligned}
$$

We employ these simple, asymptotic representations in the next section to determine the total scattering cross-section and the drag for $\kappa<\kappa_{c}$.

\section{Solution for the tall ellipses $(\epsilon \geqslant 1)$}

We pose the solution to (2.3) and (2.4) in the form

where the normalization factor

$$
\delta(\xi, \eta)=\beta \sum_{n=1}^{\infty} \gamma_{n} \delta_{n}(\xi, \eta)
$$

$$
\beta=-\frac{1}{4} \pi\left(1+\epsilon^{-1}\right) \kappa
$$

is introduced to simplify the subsequent analysis. Substituting (3.1) and (3.2) into $(2.4 b)$, we find that the $\gamma_{n}$ must be determined such that

$$
\beta \sum_{n=1}^{\infty} \gamma_{n} \delta_{n}\left(\xi_{0}, \eta\right)=\cos \eta \equiv \sum_{r=0}^{\infty} A_{1}^{2 r+1} c e_{2 r+1}(\eta)
$$

where the $A_{1}^{2 r+1}$ are the coefficients of the even Mathieu functions. Substituting (2.5) into (3.3) and equating coefficients of $c e_{2 n+1}(\eta)$ and $s e_{2 n}(\eta)$, we obtain the infinite set of linear equations

$$
\gamma_{2 n+1} M c_{2 n+1}^{(2)}+M c_{2 n+1}^{(1)} \sum_{r=1}^{\infty} \gamma_{2 r} K_{r n}=\beta^{-1} A_{1}^{2 n+1} \quad(n=0,1, \ldots)
$$

and

$$
\gamma_{2 n} M s_{2 n}^{(2)}-M s_{2 n}^{(1)} \sum_{r=0}^{\infty} \gamma_{2 r+1} K_{n r}=0 \quad(n=1,2 \ldots)
$$

where the argument of the radial Mathieu functions is $\xi_{0}$ except as explicitly noted otherwise.

Considering the complex scattering amplitude $f(\theta)$, we substitute $(2.11 b)$ and $(2.12 b)$ into (3.1) and equate the result to (1.6) to obtain

$$
f(\theta)=2 \beta \sum_{n=0}^{\infty}(-)^{n+1}\left[\gamma_{2 n+1} c e_{2 n+1}(\eta)-i \gamma_{2 n+2} s e_{2 n+2}(\eta)\right]
$$

Substituting (3.5) into (1.7a), we obtain

$$
\begin{aligned}
\frac{2 \sigma(\theta)}{\pi H \kappa^{3}\left(1+\epsilon^{-1}\right)^{2}} & \equiv \sigma_{*}(\theta) c e_{1}^{2}(\eta) \\
& =\left\{\left[\sum_{n=0}^{\infty}(-)^{n} \gamma_{2 n+1} c e_{2 n+1}(\eta)\right]^{2}+\left[\sum_{n=1}^{\infty}(-)^{n} \gamma_{2 n} s e_{2 n}(\eta)\right]^{2}\right\} .
\end{aligned}
$$

Substituting $(3.6 b)$ into $(1.7 b, c)$ we obtain

$$
\frac{8 Q}{\pi^{2} H \kappa^{3}\left(1+\epsilon^{-1}\right)^{2}} \equiv Q_{*}=\sum_{n=1}^{\infty} \gamma_{n}^{2}
$$


and $\begin{aligned} \frac{6 D}{\pi q H \kappa^{3}\left(1+\epsilon^{-1}\right)^{2}} & \equiv D_{*}=\frac{6}{\pi H \kappa^{3}\left(1+\epsilon^{-1}\right)^{2}} \int_{0}^{\frac{1}{2} \pi} \sigma(\theta) \sin \eta d \eta \\ & =\sum_{n=0}^{\infty} \sum_{p=0}^{\infty}(-)^{n+p}\left\{\gamma_{2 n+1} \gamma_{2 p+1} I_{n p}+\gamma_{2 n+2} \gamma_{2 p+2} J_{n p}\right\},\end{aligned}$

where

$$
\begin{aligned}
I_{n p} & =3 \int_{0}^{\frac{1}{2} \pi} c e_{2 n+1}(\eta) c e_{2 p+1}(\eta) \sin \eta d \eta \\
& =\frac{3}{2} \sum_{r=0}^{\infty} \sum_{s=0}^{\infty} A_{2 r+1}^{2 n+1} A_{2 s+1}^{2 p+1}\left\{\frac{1}{1-4(r-s)^{2}}+\frac{1}{1-4(r+s+1)^{2}}\right\}, \\
J_{n p} & =3 \int_{0}^{\frac{1}{2} \pi} s e_{2 n+2}(\eta) s e_{2 p+2}(\eta) \sin \eta d \eta \\
& =\frac{3}{2} \sum_{r=1}^{\infty} \sum_{s=1}^{\infty} B_{2 r}^{2 n+2} B_{2 s}^{2 p+2}\left\{\frac{1}{1-4(r-s)^{2}}-\frac{1}{1-4(r+s)^{2}}\right\},
\end{aligned}
$$

and the $B_{n}^{m}$ are the coefficients of the odd Mathieu functions. Our definition of the starred quantities and our normalization of the $\gamma_{n}$ is such that $\gamma_{1}, \sigma_{*}(\theta), Q_{*}$, and $D_{*}$ are all $I+O\left(\kappa^{2} \log \kappa\right)$ as $\kappa \rightarrow 0$.

Turning to the actual solution of the linear equations (3.4), we truncate at $n=N=1$ to obtain the first approximation

$$
\left[\sigma_{*}^{(1)}(\theta), \quad Q_{*}^{(1)}, \quad D_{*}^{(1)}\right]=\left\{\gamma_{1}^{(1)}\right\}^{2}\left[1,1, I_{00}\right],
$$

where

$$
\begin{aligned}
\gamma_{1}^{(1)} & =\beta^{-1} A_{1}^{1} / M c_{1}^{(2)} \\
& =1+\frac{1}{4}\left(1+\epsilon^{-1}\right) \kappa^{2} \log \kappa+O\left(\kappa^{2}\right) .
\end{aligned}
$$

Truncating at $n=N=2$, we obtain the second approximation

$\left[\sigma_{*}^{(2)}(\theta), \quad Q_{*}^{(2)}, \quad D_{*}^{(2)}\right]=\left\{\gamma_{1}^{(2)}\right\}^{2}\left[1+\mathscr{J}^{2}\left\{s e_{2}^{2}(\eta) / c e_{1}^{2}(\eta)\right\}, \quad 1+\mathscr{J}^{2}, \quad I_{00}+\mathscr{J}^{2} J_{00}\right]$,

where

$$
\begin{aligned}
\gamma_{1}^{(2)} & =\gamma_{1}^{(1)}\left\{1+\left(\mathscr{J} K_{10} M c_{1}^{(1)} / M c_{1}^{(2)}\right)\right\}^{-1} \\
& =\gamma_{1}^{(1)}\left\{1-\frac{1}{144} \epsilon^{-1}\left(1+\epsilon^{-1}\right)^{3} \kappa^{6}+O\left(\kappa^{8} \log \kappa\right)\right\}
\end{aligned}
$$

and

$$
\begin{aligned}
\mathscr{J} & =\gamma_{2}^{(2)} / \gamma_{1}^{(2)}=K_{10} M s_{2}^{(1)} / M s_{2}^{(2)} \\
& =\frac{1}{48} \epsilon^{-1}\left(+\epsilon^{-1}\right)^{2} \kappa^{4}+O\left(\kappa^{6}\right) .
\end{aligned}
$$

The first approximation breaks down entirely $\left(\gamma_{1}^{(1)}=\infty\right)$ at the smallest positive value of $\kappa$, say $\kappa_{1}$, for which $M c_{1}^{(2)}\left(\xi_{0}, \frac{1}{4} \omega^{2}\right)$ vanishes. We can show that the $N$ 'th approximation breaks down at $\kappa=\kappa_{N}$, where $\kappa_{N}$ is approximately the smallest positive value of ' $\kappa$ for which $M c_{N}^{(2)}\left(\xi_{0}, \frac{1}{4} \omega^{2}\right)$ vanishes. Examining the curves of $M c_{n}^{(2)}(\xi, s), n=0,1,2$, presented by Wiltse \& King (1958a)† and recalling the interlace theorem for the zeros of $Y_{n}(z)$, the corresponding functions

† The tables of Wiltse \& King (1958 a,b) [see Blanch (1959) for a summary of both sets of these tables] appear to be the only ones that tabulate both kinds of radial Mathieu functions. They are not, however, adequate for the present numerical investigation, which was performed on a CDC 3600. 
for $\varepsilon=1$, we conjecture that $\kappa_{N}$ increases monotonically with $N$ (for fixed $\epsilon$ ). We find that $\kappa_{1}$ is considerably larger than $\kappa_{c}$.

Numerical investigation shows that the difference between successive approximations is largest for $\epsilon=1$. We find that the first approximation is adequate for reasonably small $\kappa ; D_{*}^{(1)}$ differs from $D_{*}^{(2)}$ by at most $1 \%$ at $\kappa=0.75$ but departs rapidly therefrom for larger $\kappa(5 \%$ at $\kappa=1.0$, a maximum of $18 \%$ at $\left.\kappa=\kappa_{c}\right)$. The third approximation to $D_{*}$ differs from the second by less than $0.2 \%$ at $\kappa=\kappa_{c}$ and approximately $1 \%$ at $\kappa=2 \cdot 0$. We find the second approximation entirely adequate for numerical purposes.

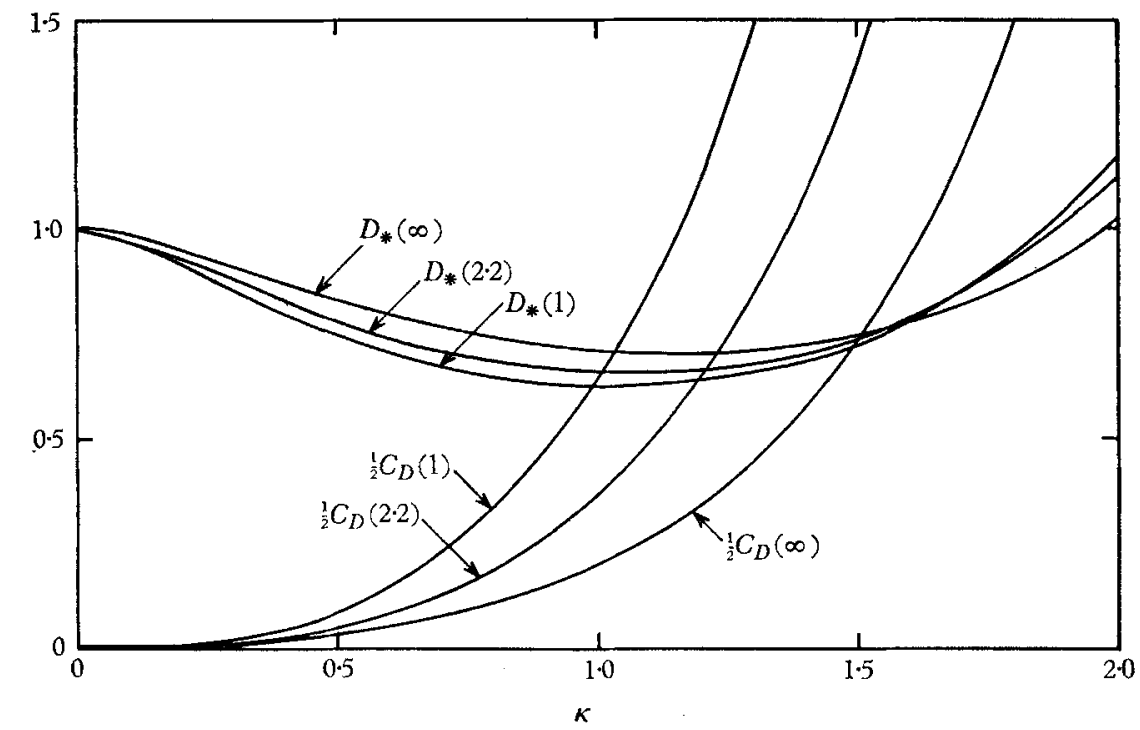

FiauRe 2. Normalized drag $\left(D_{*}\right)$ and drag coefficient $\left(C_{D}\right)$ for three tall ellipses.

Considering the limit $\kappa \rightarrow 0$ in (3.4), we find that

$$
\gamma_{2 n}, \gamma_{2 n+1}=O\left(\kappa^{4 n}\right) \quad(n=1,2, \ldots) \quad(\kappa \rightarrow 0)
$$

unless $\epsilon=1$, in which case $\gamma_{2 n+1}=O\left(\kappa^{4 n+6}\right)$. In particular, we infer from the third approximation (not given explicitly here because it is algebraically unwieldy) that $\sigma_{*}(\theta), Q_{*}$, and $D_{*}$ differ from one another by $O\left(\kappa^{4}\right)$ unless $\varepsilon=1$, in which case they differ by $O\left(\kappa^{8}\right)$. We also can prove that the error factor associated with the first approximation is $O\left(\kappa^{4}\right)$ unless $\epsilon=1$, in which case it is $O\left(\kappa^{6}\right)$.

Setting $\xi_{0}=0$ (thin barrier) in (3.4) and recalling that $M s_{n}^{(\mathbf{1})}\left(0, \frac{1}{4} \omega^{2}\right)=0$, we find that the infinite set of equations (3.4) is no longer coupled and has a solution equivalent to that given by $I(6.14 a)$. The value of $\kappa_{c}$, as reported in $I$, is $1 \cdot 73$. The results for $D_{*}$ and the drag coefficient

$$
C_{D}=D / q H
$$

are presented in figure 2. The streamline pattern for $\kappa=\kappa_{c}$ is presented in figure 3 . [All figures depicting streamlines, in both II and this paper, are scaled such that the various obstacles are of equal height.]

The semi-circular obstacle is described by $\epsilon=1$, which implies $\xi_{0}=\infty$. Letting $\xi_{0} \rightarrow \infty$ in (3.2)-(3.16), we recover the results given in II, $\S 3$. The value of $\kappa_{c}$, as 


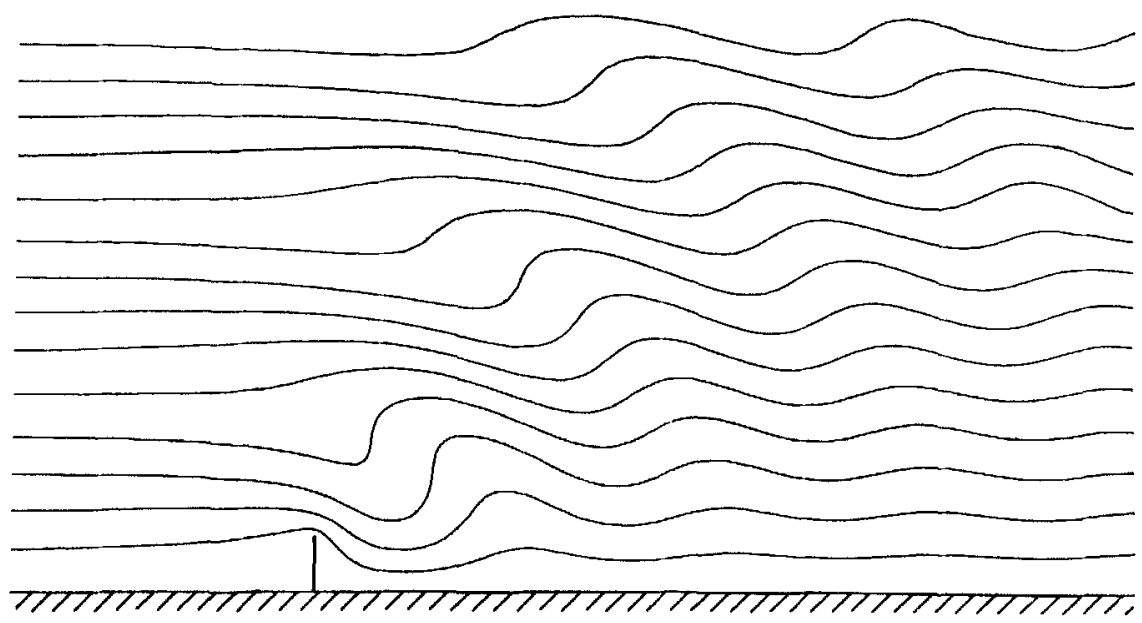

Figure 3. Stratified shear flow over a thin barrier for $\kappa=\kappa_{c}=1 \cdot 73$.

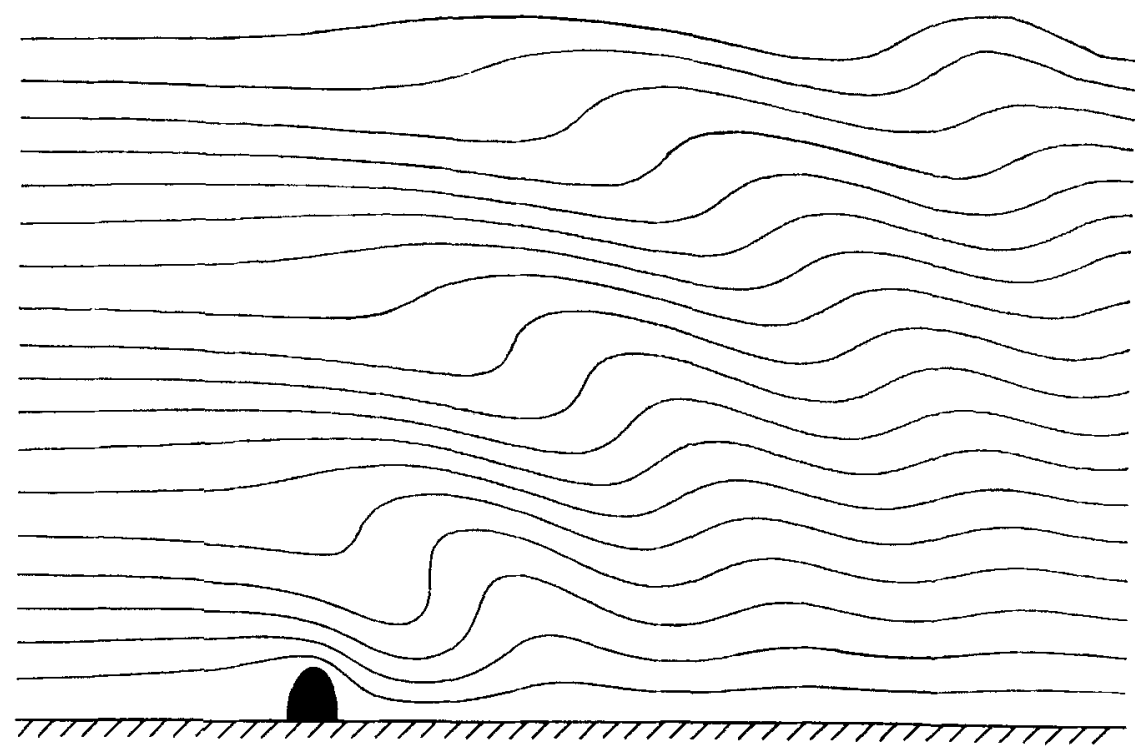

Figure 4. Stratified shear flow over the semi-elliptical obstacle $\epsilon=2 \cdot 2$ for $\kappa=\kappa_{c}=1 \cdot 5$. 
reported in II, is $1 \cdot 27$. The results for $D_{*}$ and $C_{D}$, also reported in II, are reproduced in figure 2 .

The values of $Q_{*}$ and $D_{*}$ for ellipses with $1<\epsilon<\infty$ are similar to those already presented for $\epsilon=1, \infty$. We find that for $1 \leqslant \epsilon<\infty$ and $\kappa<1.7$ the variation in $D_{*}, q u a$ function of $\epsilon$, is less than $15 \%$ and that $Q_{*}$ is less than or equal to $D_{*}$ and differs from $D_{*}$ by at most $12 \%$. We present $D_{*}$ and the drag coefficient for a representative ellipse, $\epsilon=2 \cdot 2$, in figure 2 . The streamlines for this elliptical obstacle for $\kappa=\kappa_{c}=1.5$ are plotted in figure 4. Examining the curves of $C_{D}$ against $\kappa$ for various values of $\epsilon$, we find that the maximum drag coefficient (the drag coefficient at $\kappa=\kappa_{c}$ ) increases from $2 \cdot 3$ to $2 \cdot 8$ as $\epsilon$ decreases from infinity to one.

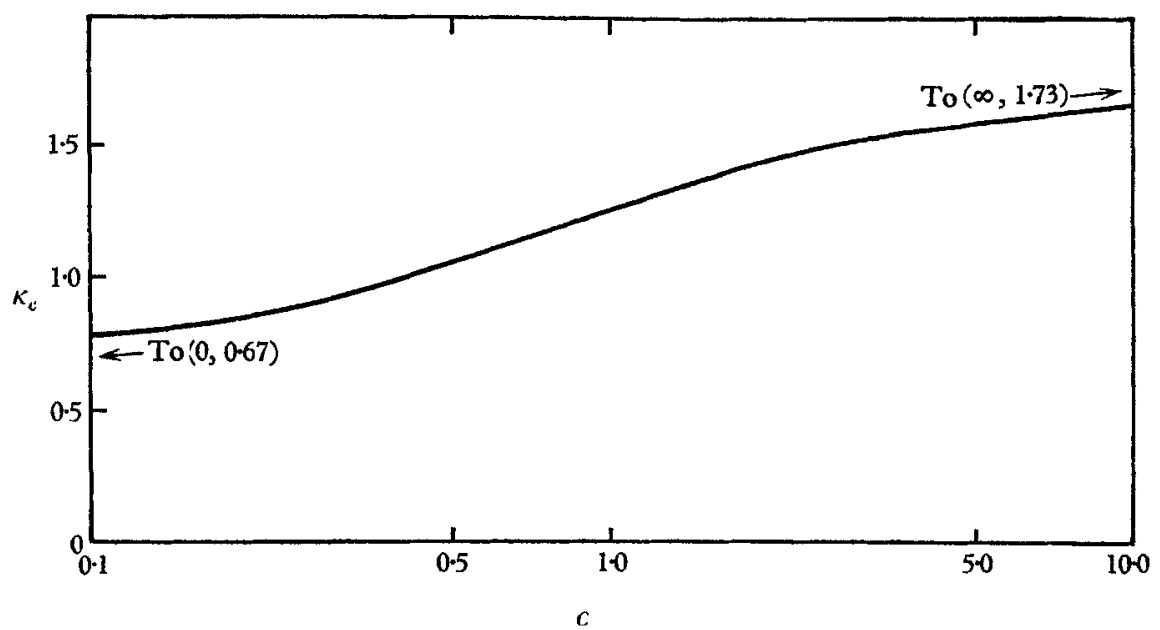

Frgure 5. The reduced frequency at which static instability first occurs.

Considering the stability of the flow $\left(\delta_{y}<1\right.$ everywhere for $\kappa<\kappa_{c}$ ), we find that, as $\epsilon$ decreases from infinity to one, $\kappa_{c}$ decreases monotonically from 1.73 (thin barier) to 1.27 (semi-circle). We present $\kappa_{c}$ as a function of $\epsilon$ in figure 5 .

\section{The squat ellipses $(\epsilon \leqslant 1)$}

We abbreviate the following analysis in recognition of the fact that the mathematical details for the squat ellipses are similar to those for the tall ellipses. We define the variables $\xi, \hat{\eta}, \hat{\omega}, \hat{h}$ by

$$
\begin{gathered}
x=\hat{h} \cosh \xi \cos \hat{\eta}, \quad y=\hat{h} \sinh \xi \sin \hat{\eta}, \\
\hat{\omega}=\kappa \hat{h}=(N H / U)\left\{(B / H)^{2}-1\right\}^{\frac{1}{2}}, \quad h=\epsilon^{-1}\left(1-\epsilon^{2}\right)^{\frac{1}{2}}=\left\{(B / H)^{2}-1\right\}^{\frac{1}{2}} .
\end{gathered}
$$

These variables are related to those defined in $(2.1)$ by

$$
\begin{array}{cl}
\xi=\xi-\frac{1}{2} i \pi, & \eta=\frac{1}{2} \pi-\hat{\eta}, \\
\omega=i \hat{\omega}, & h=i \hat{h} .
\end{array}
$$

The obstacle is now specified by

$$
\xi=\xi_{0} \equiv \tanh ^{-1} \epsilon \text {. }
$$


Substituting the definitions (4.1)-(4.3) into (1.2) and (1.4) and dropping the hats, we obtain

$$
\begin{gathered}
\delta_{\xi \xi}+\delta_{\eta \eta}+\omega^{2}\left(\sinh ^{2} \xi+\sin ^{2} \eta\right) \delta=0, \\
\delta(\xi, 0)=\delta(\xi, \pi)=0 \quad\left(\xi>\xi_{0}\right), \\
\delta\left(\xi_{0}, \eta\right)=\sin \eta,
\end{gathered}
$$

and

$$
\delta(\xi, \eta)=o\left(\exp \left[-\frac{1}{2} \xi\right]\right) \quad\left(\xi \rightarrow \infty, \frac{1}{2} \pi<\eta<\pi\right) .
$$

We find that an appropriate set of lee-wave functions, which satisfies (4.4) and $(4.5 a, b, d)$ and is complete in $0<\eta<\pi$ for fixed $\xi$, is given by

where

$$
\delta_{n}(\xi, \eta)=M s_{n}^{(2)}(\xi) s e_{n}(\eta)+\psi_{n}(\xi, \eta) \quad(n=1,2, \ldots),
$$

$$
\begin{aligned}
\psi_{n}(\xi, \eta) & =\sum_{r=1}^{\infty} D_{n r} M s_{r}^{(1)}(\xi) s e_{r}(\eta) \\
= & \pi^{-1}(i)^{n} \int_{0}^{\pi} \operatorname{sgn}\left(\frac{1}{2} \pi-t\right) e^{-i \omega \cosh \xi \cos \eta \cos t} \sin (\omega \sinh \xi \sin \eta \sin t) s e_{n}(t) d t \\
D_{n r} & =F_{n r} \quad(n \text { odd, } \quad r \text { even }) \\
& =-F_{r n} \quad(n \text { even, } \quad r \text { odd }) \\
& =0 \quad(n-r \text { even }),
\end{aligned}
$$

and

$$
F_{n r}=\frac{1}{4} \omega^{3} g_{o, n} g_{o, r} B_{1}^{n} B_{2}^{r} /\left(b_{r}-b_{n}\right) .
$$

We again suppress the argument $\frac{1}{4} \omega^{2}$ from the Mathieu functions. Each member of the set (4.6) satisfies (4.5d) and has the asymptotic form

$$
\begin{aligned}
\delta_{n}(\xi, \eta) & \sim 2 M s_{n}^{(2)}(\xi) s e_{n}(\eta) \quad\left(\omega \cosh \xi \rightarrow \kappa r \rightarrow \infty, \quad 0 \leqslant \eta<\frac{1}{2} \pi\right) \\
& \sim(-)^{n} 2^{\frac{3}{2}}(\pi \omega \cosh \xi)^{-\frac{1}{2}} \sin \left(\omega \cosh \xi+\frac{1}{2} n \pi-\frac{1}{4} \pi\right) e_{n}(\eta) \quad(\xi \rightarrow \infty) .
\end{aligned}
$$

We pose the solution to (4.4), (4.5) in the form

where

$$
\delta(\xi, \eta)=\beta \sum_{n=1}^{\infty} \gamma_{n} \delta_{n}(\xi, \eta)
$$

and the $\gamma_{n}$ are to be determined such that

$$
\beta \sum_{n=1}^{\infty} \gamma_{n} \delta_{n}\left(\xi_{0}, \eta\right)=\sin \eta \equiv \sum_{r=0}^{\infty} B_{1}^{2 r+1} s e_{2 r+1}(\eta) .
$$

Proceeding as in $\$ 3$, we obtain the infinite set of linear equations

$$
\gamma_{n} M s_{n}^{(2)}+M s_{n}^{(1)} \sum_{r=1}^{\infty} \gamma_{r} D_{r n}=\beta^{-1} B_{1}^{n} \quad(n=1,2, \ldots)
$$

where

$$
B_{1}^{2 s} \equiv 0 \quad(s=1,2, \ldots)
$$

and

$$
M s_{n}^{(j)} \equiv M s_{n}^{(j)}\left(\xi_{0}, \frac{1}{4} \omega^{2}\right) \quad(j=1,2) .
$$

$\dagger$ Merbt (1959) considered the set of equations (4.4), $(4.5 a, b, d)$ and $\delta(0, \eta)=s e_{2}(\eta)$. These equations result from Lyra's (1943) model for lee-wave formation. Merbt posed an expansion similar to (4.6) but determined the coefficients of $\delta_{2}$ (the only mode required) approximately. His is an inverse method of solution in the sense that his obstacle is a function of the reduced frequency. There is no significant overlap with our work. 
Calculating the complex scattering amplitude, the differential scattering crosssection, the total scattering cross-section, and the drag, we obtain

$$
\begin{aligned}
& f(\theta)=2 \beta \sum_{n=0}^{\infty}(-)^{n+1}\left[\gamma_{2 n+1} s e_{2 n+1}(\eta)-i \gamma_{2 n+2} s e_{2 n+2}(\eta)\right], \\
& \frac{2 \sigma(\theta)}{\pi H \kappa^{3} \epsilon^{-2}(1+\epsilon)^{2}} \equiv \sigma_{*}(\theta) s e_{1}^{2}(\eta) \\
&=\left\{\left[\sum_{n=0}^{\infty}(-)^{n} \gamma_{2 n+1} s e_{2 n+1}(\eta)\right]^{2}+\left[\sum_{n=1}^{\infty}(-)^{n} \gamma_{2 n} s e_{2 n}(\eta)\right]^{2}\right\}, \\
& \overline{\pi^{2}} \frac{8 Q}{H \kappa^{3} \epsilon^{-2}(1+\epsilon)^{2}} \equiv Q_{*}=\sum_{n=1}^{\infty} \gamma_{n}^{2},
\end{aligned}
$$

and

$$
\begin{aligned}
\frac{6 D}{\pi q H \kappa^{3} \epsilon^{-2}(1+\epsilon)^{2}} \equiv D_{*} & =\frac{6}{\pi H \kappa^{3} \epsilon^{-2}(1+\epsilon)^{2}} \int_{0}^{\frac{1}{2} \pi} \sigma(\theta) \cos \eta d \eta \\
& =\sum_{n=0}^{\infty} \sum_{p=0}^{\infty}(-)^{n+p}\left\{\gamma_{2 n+1} \gamma_{2 p+1} S_{n p}+\gamma_{2 n+2} \gamma_{2 p+2} T_{n p}\right\}
\end{aligned}
$$

and

$$
\begin{aligned}
T_{n p} & =3 \int_{0}^{\frac{1}{2} \pi} s e_{2 n+2}(\eta) s e_{2 p+2}(\eta) \cos \eta d \eta \\
& =\frac{3}{2} \sum_{r=1}^{\infty} \sum_{s=1}^{\infty}(-)^{r+s} B_{2 r}^{2 n+2} B_{2 s}^{2 p+2}\left\{\frac{1}{1-4(r-s)^{2}}-1 \frac{1}{1-4(r+s)^{2}}\right\} .
\end{aligned}
$$

Turning to the actual solution of (4.13) and the evaluation of the scattering cross-sections, drag, and instability point, we truncate (4.13) at $n=N=1$ to obtain the first approximation

$$
\left[\sigma_{*}^{(1)}(\theta), \quad Q_{*}^{(1)}, \quad D_{*}^{(1)}\right]=\left\{\gamma_{1}^{(1)}\right\}^{2}\left[1,1, S_{00}\right],
$$

where

$$
\begin{aligned}
\gamma_{1}^{(1)} & =\beta^{-1} B_{1}^{1} / M s_{1}^{(2)} \\
& =1+\frac{1}{4} \epsilon^{-1}(1+\epsilon) \kappa^{2} \log \kappa+O\left(\kappa^{2}\right) .
\end{aligned}
$$

Truncating at $n=N=2$, we obtain the second approximation

$$
\begin{aligned}
& {\left[\sigma_{*}^{(2)}(\theta), \quad Q_{*}^{(2)}, \quad D_{*}^{(2)}\right]=\left\{\gamma_{1}^{(2)}\right\}^{2}\left[1+\mathscr{G}^{2}\left\{s e_{2}^{2}(\eta) / s e_{1}^{2}(\eta)\right\}, \quad 1+\mathscr{G}^{2}, \quad S_{00}+\mathscr{G}^{2} T_{00}\right]} \\
& \text { where } \\
& \gamma_{1}^{(2)}=\gamma_{1}^{(1)}\left\{1+\left(\mathscr{G} F_{12} M s_{1}^{(1)} / M s_{1}^{(2)}\right)\right\}^{-1} \\
& =\gamma_{1}^{(1)}\left\{1-\frac{1}{14} \epsilon^{-4}(1+\epsilon)^{3} \kappa^{6}+O\left(\kappa^{8} \log \kappa\right)\right\} \\
& \mathscr{G}=-\gamma_{2}^{(2)} / \gamma_{1}^{(2)}=F_{12} M s_{2}^{(1)} / M s_{2}^{(2)} \\
& =-\frac{1}{48} \epsilon^{-3}(1+\epsilon)^{2} \kappa^{4}+O\left(\kappa^{6}\right) \text {. }
\end{aligned}
$$

Our procedure now departs from that of $\S 3$. We find that two terms in the expansion (4.11) do not suffice for small $\epsilon$. The number of terms required increases with the parametric argument of the Mathieu functions

$$
\frac{1}{4} \omega^{2}=\frac{1}{4}\left(\epsilon^{-2}-1\right) \kappa^{2}
$$


which tends to infinity as $\epsilon \rightarrow 0$ for fixed $\kappa$. For $\epsilon=0 \cdot 3, D_{*}^{(3)}$ is less than $D_{*}^{(2)}$ by $10 \%$ at $\kappa=\kappa_{c}=0.93$. For $\epsilon=0.1, D_{*}^{(3)}$ is less than $D_{*}^{(2)}$ by $13 \%$ at $\kappa=0.6$ $\left(\kappa_{c}=0 \cdot 78\right)$.

The linearized solution to (4.4) and (4.5) yields (IV, $\S 7)$

$$
\begin{aligned}
D_{*} & =6 k^{-3}\left[k+J_{1}(2 k)-\int_{0}^{2 k} J_{0}(x) d x\right] \quad(\epsilon \rightarrow 0) \\
& =1-\frac{1}{10} k^{2}+O\left(k^{4}\right) \quad(\epsilon \rightarrow 0, \quad k \rightarrow 0),
\end{aligned}
$$

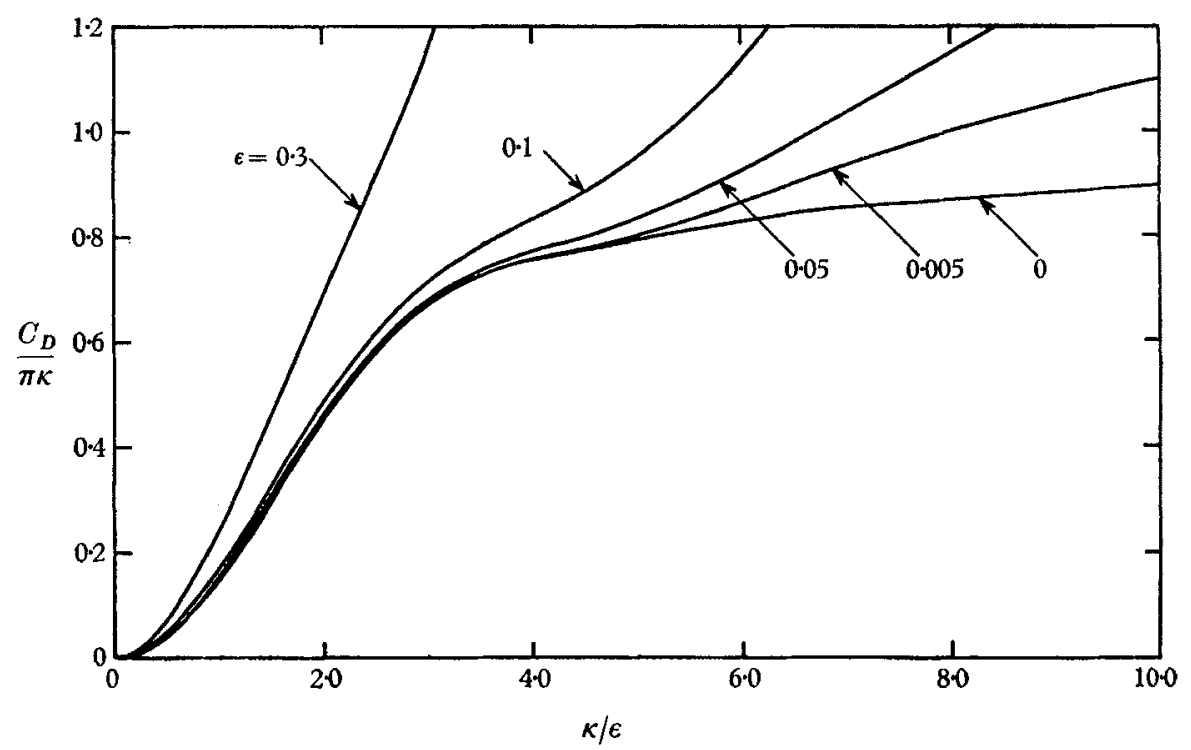

Figure 6. Normalized drag coefficient for squat ellipses with small $\epsilon$.

where

$$
k=\kappa / \epsilon .
$$

We use (4.29) to determine the accuracy of the solution obtained by truncating (4.11) at $n=N=3$.

We present $C_{D} / \pi \kappa$, qua function of $k$, for various values of $\epsilon$ in figure 6 . 'This plot gives some indication of the domains of validity of the third approximation and the linearized solution. We develop the solution for $\epsilon \ll 1$ and $k \gg 1$ in IV $(\$ 5)$, which covers the domain for which the expansion (4.11) is not satisfactory.

The approximate solutions to (4.13) break down in a manner entirely similar to that described in $\S 3$, we need only replace $M c_{N}^{(2)}\left(\xi_{0}, \frac{1}{4} \omega^{2}\right)$ by $M s_{N}^{(2)}\left(\xi_{0}, \frac{1}{4} \omega^{2}\right)$. We mention explicitly only that $\kappa_{1}$, the value of $\kappa$ at which the first approximation breaks down, is always larger than $\kappa_{c}$. Truncating (4.13) at $n=N=3$, we infer that $\sigma_{*}(\theta), Q_{*}$, and $D_{*}$ differ from one another by $O\left(\kappa^{4}\right)$ for $\epsilon<1$ and that the error factor associated with the first approximation is $O\left(\kappa^{4}\right)$. For $\epsilon=1,(4.13)$ reduces to the equations given by II (3.4).

Using the third approximation, we present $D_{*}$ and $C_{D}$ in figures 7 and 8 . We remark that $D_{*}$, qua function of $\epsilon$ for the squat ellipses, varies by orders of magnitude. This constrasts with the $15 \%$ variation determined for the tall ellipses. We also find that $Q_{*}$ for the squat ellipses may be either greater or less than $D_{*}$ and 


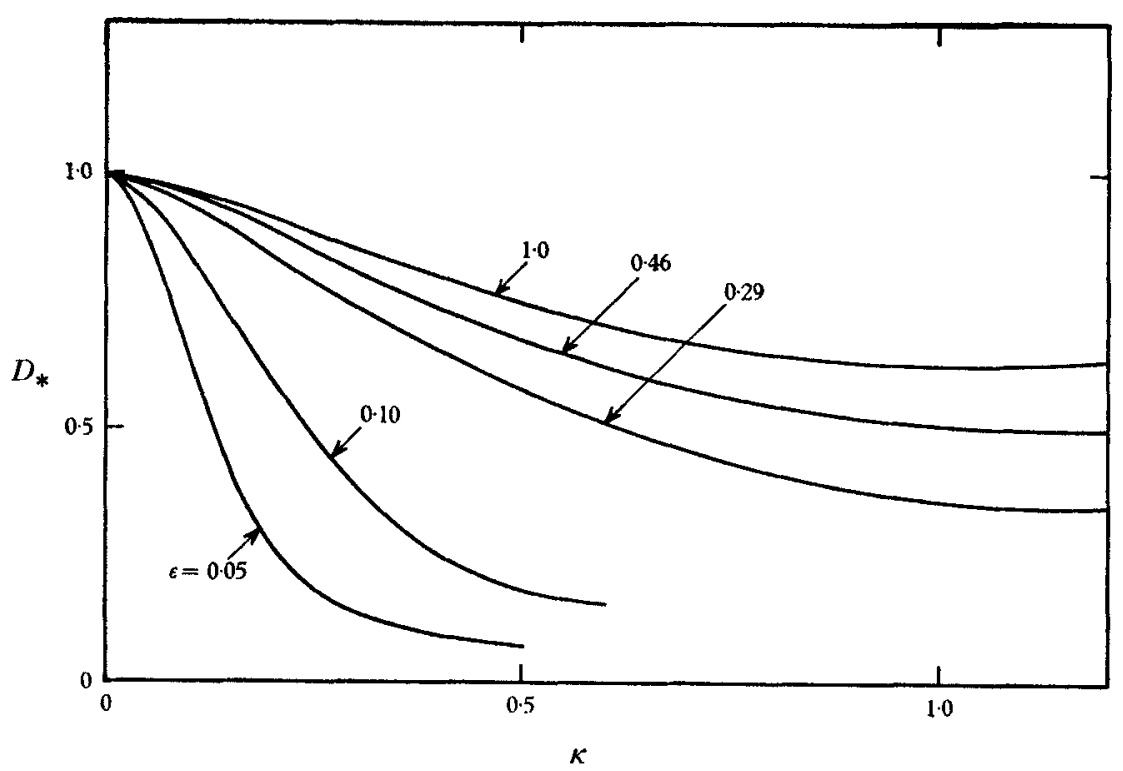

Figure 7. Normalized drag for squat ellipses.

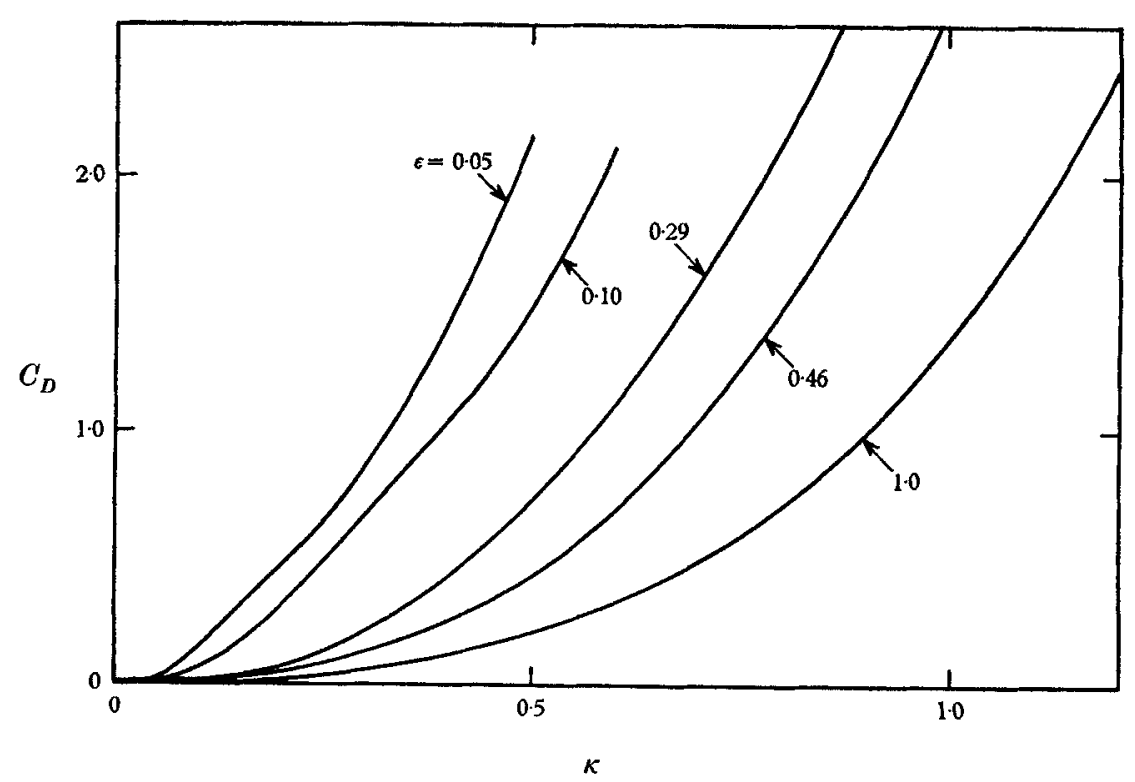

Figure 8. Drag coefficient for squat ellipses. 


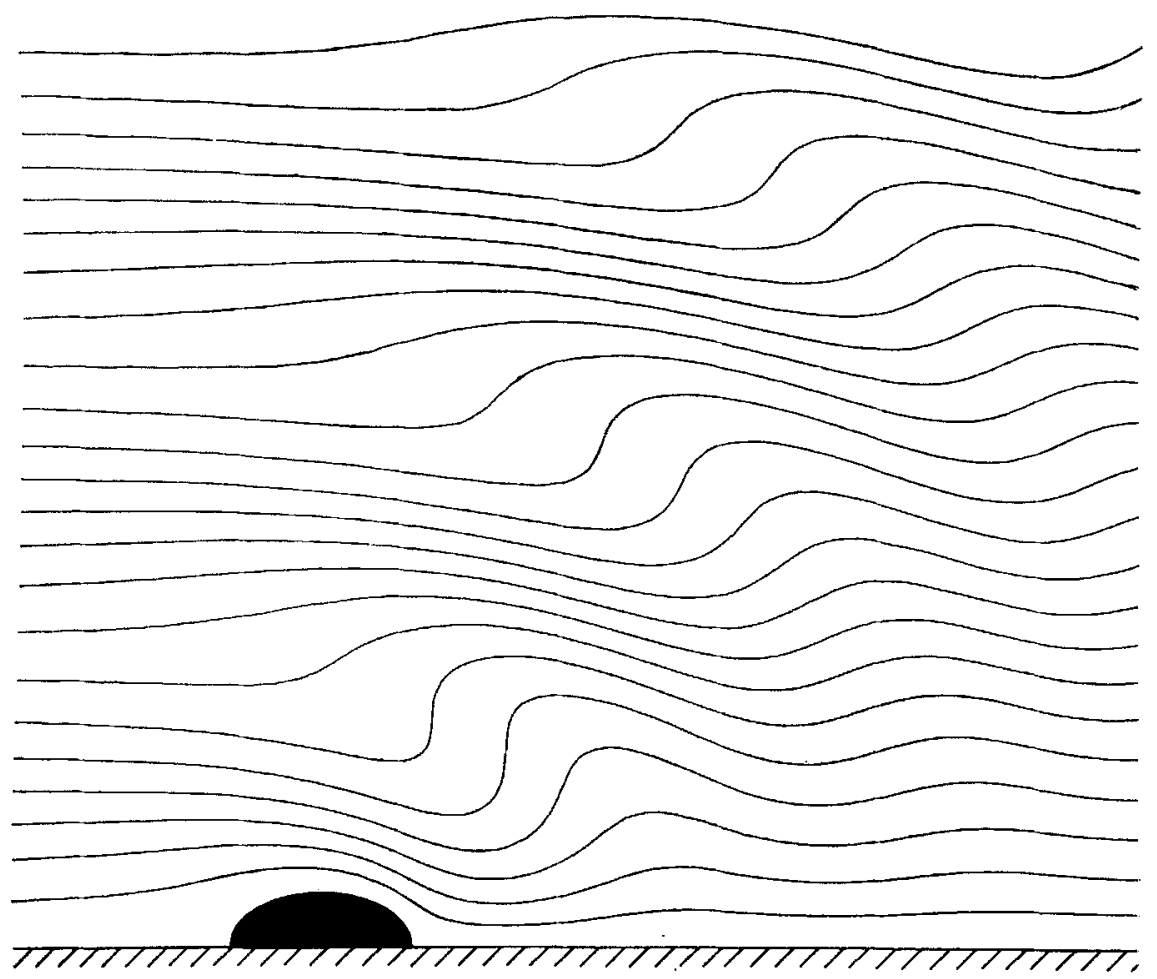

Figure 9. Stratified shear flow over the semi-elliptical obstacle $\epsilon=0 \cdot 6$ for $\kappa=\kappa_{c}=1 \cdot 12$.

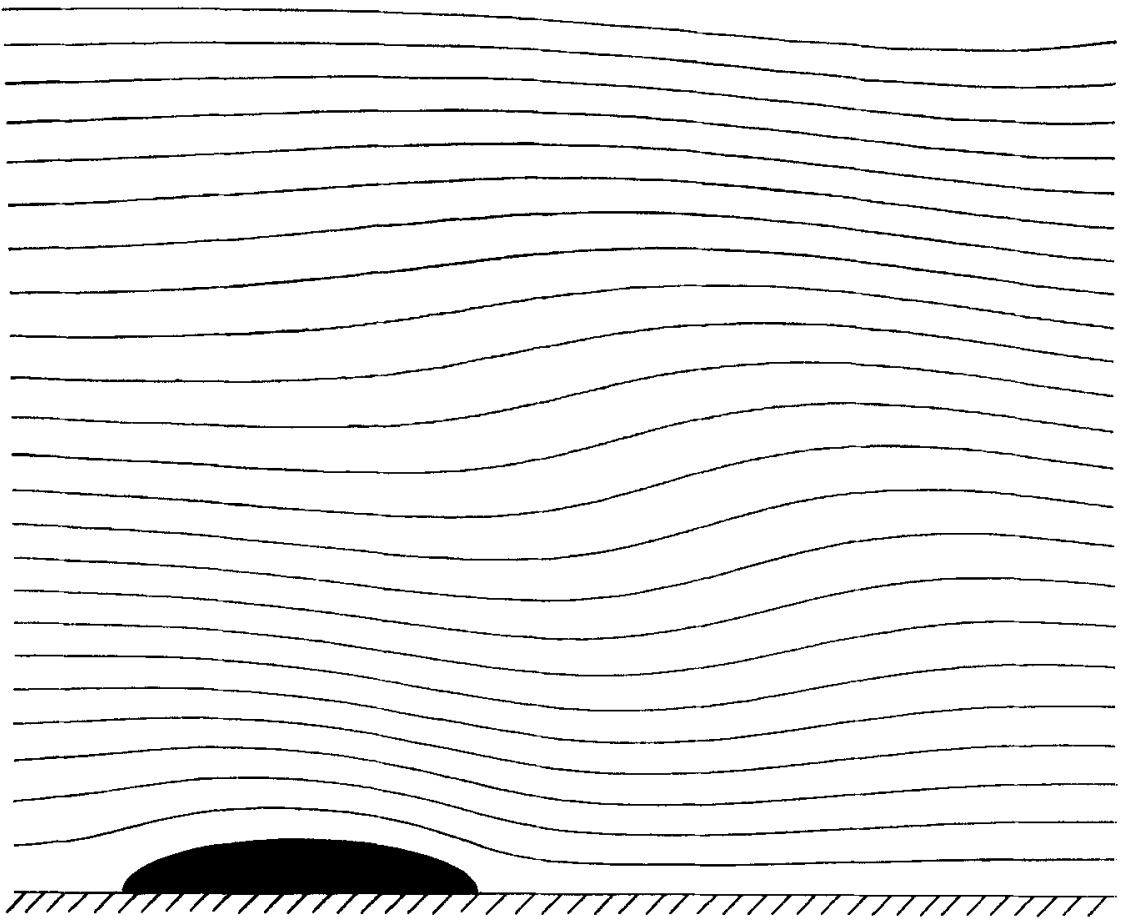

Figure 10. Stratified shear flow over the semi-elliptical obstacle $\epsilon=0.3$ for $\kappa=0.5$. 
may differ from $D_{*}$ by as much as a factor of ten. We present $\kappa_{c}$, which is monotonically increasing, in figure 5. From this figure and the values of $C_{D}$, we find that the maximum $C_{D}$ for stable flow is approximately $3 \cdot 0$ for all squat ellipses. We present the flow pattern for various values of $\epsilon$ and $\kappa$ in figures 9-11.

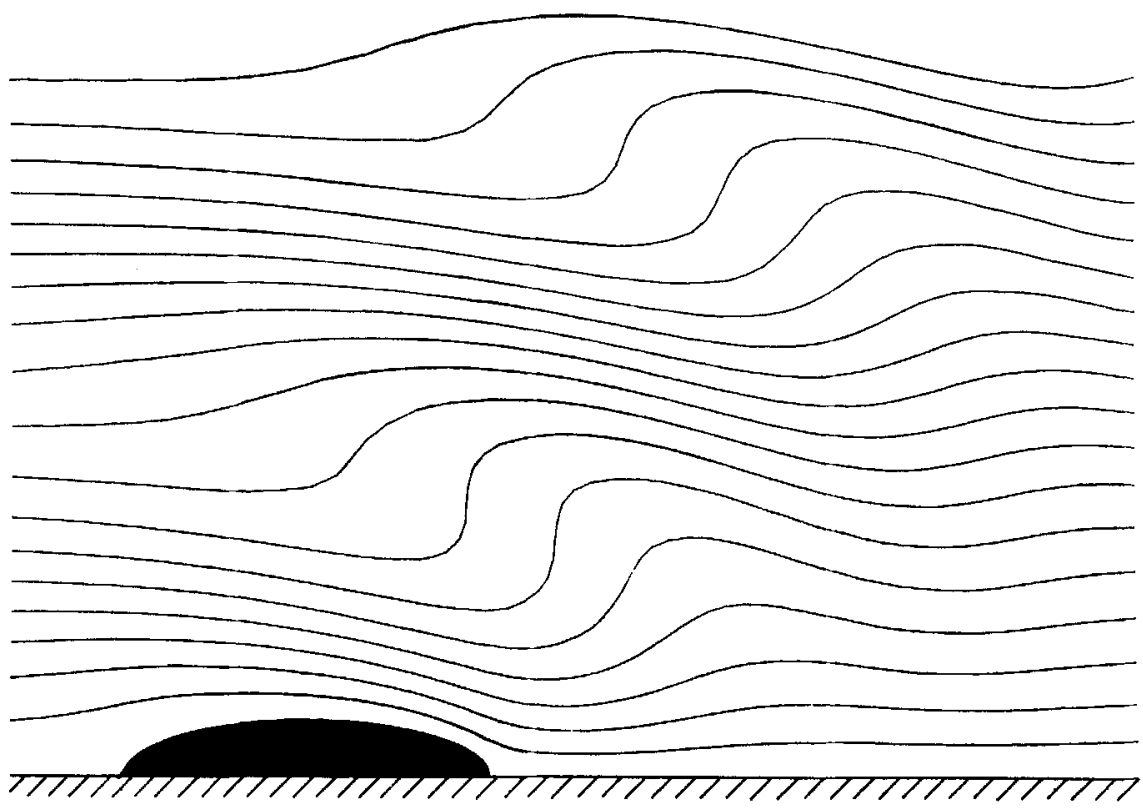

Frgure 11. Stratified shear flow over the semi-elliptical obstacle $\epsilon=0 \cdot 3$ for $\kappa=\kappa_{c}=0 \cdot 93$.

\section{Conclusions}

The maximum reduced frequency for static stability of the lee-wave field excited by a semi-elliptical obstacle of fixed height in a stratified shear flow decreases with increasing width. The maximum drag coefficient for stable, unseparated flow over a family of ellipses of constant height increases from $2 \cdot 3$ to 3.0 as the slenderness ratio decreases from infinity to zero. The amplitudes of the lee waves excited by a semi-elliptical obstacle gradually decrease with increasing altitude. The scale of the lee-wave field decreases with decreasing reduced frequency for fixed slenderness ratio and decreases with increasing slenderness ratio for fixed reduced frequency.

This research was supported in part by National Science Foundation Grant GA-849, the Office of Naval Research Contract Nonr-2216(29), and by a Sydney University Post-Graduate Travelling Fellowship (H. E. H.).

\section{REFERENCES}

Blanch, G. 1959 Math. Tables and other aids to Computation (now, Math. of Computations), $13,131-3$.

BLANCH, G. 1964 Mathieu Functions, chapter 20 in Handbook of Mathematical F'unctions (edited by M. Abramowitz and I. Stegun) Washington: National Bureau of Standards. 
LoNG, R. R. 1953 Some aspects of the flow of stratified fluids. I. A theoretical investigation. Tellus, 5, 42-58.

LyrA, G. 1943 Theorie der stationären Leewellenströmung in freier Atmosphäre. $Z$. Angew. Math. Mech. 23, 1-28.

MERBT, H. 1959 Solution of the two-dimensional lee-wave equation for arbitrary mountain profiles, and some remarks on the horizontal wind component in mountain flow. Beitr. Phys. Atmos. 31, 152-61.

Mures, J. W. 1968 a Lee waves in a stratified flow. Part 1. Thin barrier. J. Fluid Mech. 32, $549-67$.

Mrtes, J. W. $1968 b$ Lee waves in a stratified flow. Part 2. Semi-circular obstacle. J. Fluid Mech. 33, 803-14.

Mires, J. W. \& Hupperi, H. E. 1969 Lee waves in a stratified flow. Part 4. Perturbation approximations $J$. Fluid Mech. 35, 497.

Wrltse, J. C. \& King, Marcia J. 1958a Values of the Mathieu functions. Johns Hopkins University Radiation Laboratory Technical Report no. AF-53, Baltimore, Maryland.

Wintse, J. C. \& King, Marcia J. $1958 b$ Derivatives, zeros, and other data pertaining to Mathieu functions. Johns Hopkins University Radiation Laboratory Technical Report No. AF-57, Baltimore, Maryland. 